\title{
MicroRNA-200c-3p Negatively Regulates ATP2A2 and Promotes the Progression of Papillary Thyroid Carcinoma
}

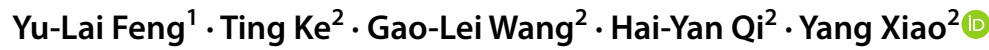

Received: 15 September 2021 / Accepted: 5 January 2022 / Published online: 25 January 2022 (C) The Author(s), under exclusive licence to Springer Science+Business Media, LLC, part of Springer Nature 2022

\begin{abstract}
microRNA-200c-3p (miR-200c-3p) has emerged as an important tumor growth regulator. However, its function in papillary thyroid carcinoma (PTC) is poorly understood. This study was conducted to investigate the role of miR-200c-3p in the progression of human PTC. The miR-200c-3p expression in human PTC tissues and cell lines was evaluated. The target relationship between miR-200c-3p and candidate genes was predicted through bioinformatic analysis and confirmed with a luciferase reporter assay. miRNA or gene expression was altered using transfection, and cell behavior was analyzed using CCK-8, wound healing, Transwell, and colony formation assays. The tumor-promoting effects of miR-200c-3p were evaluated by xenografting tumors with $\mathrm{K} 1$ cells in nude mice. The expression level of miR-200c-3p in human PTC tissues and cell lines markedly increased, and this increased expression was significantly associated with a worse overall survival. When inactivated, miR-200c-3p suppressed K1 cells' malignant behaviors, including decreasing proliferation and attenuating colony formation, migration, and invasion. Its inactivation also attenuated the development of xenografted $\mathrm{K} 1$ cells in nude mice. The effects of miR-200c-3p mimics on promoting the malignant behaviors of PTC cells were remarkably reversed by the overexpression of ATP2A2, as a downstream target of miR-200c-3p. miR-200c-3p acts as an oncogenic gene and promotes the malignant biological behaviors of human PTC cells, thereby directly targeting ATP2A2. This regulated axis may be used as a potential therapy of PTC.
\end{abstract}

Yu-Lai Feng and Ting Ke have contributed equally to this work.

Hai-Yan Qi

future1058@qq.com

$\triangle$ Yang Xiao

xyangdoc@126.com

Extended author information available on the last page of the article 
Keywords microRNA $\cdot$ Papillary thyroid carcinoma $\cdot$ miR-200c-3p $\cdot$ ATP2A2 ·

Proliferation · Metastasis

\section{Introduction}

The incidence of thyroid cancer, a common endocrine cancer, increases yearly (Vecchia et al. 2015). For instance, about 52,070 new cases were reported in the United States in 2019, and nearly 2,170 patients died of thyroid cancer (Siegel et al. 2019). Follicular cells in the thyroid can undergo neoplastic transformation. The well-differentiated type of malignancies in follicular cells can be further subdivided into two subtypes: papillary thyroid carcinoma (PTC) and follicular thyroid carcinoma (FTC). PTC originates de novo and is the most common type of thyroid cancer (80\%-85\%; Sipos and Mazzaferri 2010; Xing 2013). It is characterized by multifocality, lymphatic or local spreading, and lymph node metastasis, and the overall 5-year survival rate of patients with PTC is about $97 \%$. It is usually treated with radioiodine ablation therapy during and after surgery for most patients with low-risk small tumors and those who have no local/distant metastases or extra-thyroidal infiltration (Coca-Pelaz et al. 2020; Limaiem et al. 2021). However, some patients with metastatic, recurrent, and relapsed PTC require prompt and comprehensive treatments. Therefore, the molecular mechanisms underlying PTC disease progression should be elucidated to identify new therapeutic targets and develop more effective PTC therapeutic strategies.

MicroRNAs (miRNAs) are small noncoding RNAs encoded by 19-25 nucleotides. They control gene expression in a post-transcriptional manner, thereby influencing diverse biological activities. In general, miRNAs reduce the expression of their target genes by promoting the instability of their transcript and suppressing the translation of targets (Ambros 2004; Bartel 2004). They fundamentally modulate various physiological processes, such as cancer and tumorigenesis progression, by downregulating the abundance of specific proteins in these manners (Ghafouri-Fard et al. 2020). The adapted expression of miRNAs has also been identified in many studies on PTCs. For example, He et al. (2005) identified five miRNAs, namely, miR-146, miR-221, miR-222, miR-21, and miR-181a, and compared them with normal thyroid tissues. They found that these miRNAs are significantly overexpressed in PTCs. Further studies have suggested that Mothers against decapentaplegic homolog 4(SMAD4)is a critical member of the transforming growth factor $\beta$ (TGF- $\beta$ ) signaling pathway; it functions as an hsamiR146b (a miR-146 family member) target, which confers the ability of hsamiR146b in controlling PTC cell proliferation (Geraldo et al. 2012). In addition, hsa-miR146b-5p increases the expression of frizzled class receptor 6 and LDL receptor-related protein 6 by inhibiting zinc ring finger3; consequently, Wnt/ $\beta$ catenin signaling increases, and alternating epithelial-mesenchymal transition (EMT) occurs in PTCs (Deng et al. 2015). Therefore, miRNAs are efficient biomarker candidates and promising therapeutic tools for PTCs.

MiR-200c-3p, a key member of miR-200 family, a cancer-related miRNA belonging to the miR-200 family, can regulate epithelial-mesenchymal transition 
(EMT) in cells or tissues (Chen et al. 2021; Mao and Jiang 2021; Pontemezzo et al. 2021) and modulating tumor proliferation, invasion and metastasis (Wang et al. 2020, 2021; Xia et al. 2020). Up to date, miR-200c-3p has been widely reported as a tumor-suppressive or an oncogenic miRNA in many cancer types. For instance, miR-200c-3p was downregulated in cancer tissues and inhibited the proliferation, EMT, migration, and invasion and induced the apoptosis and chemosensitivity in non-small cell lung cancer (Wang et al. 2020), prostate cancer (Xia et al. 2020), and breast cancer (Chen et al. 2021), while recent studies have conversely reported that miR-200c-3p was increased in cancer tissue compared with the normal tissues adjacent to cancer and promoted cell viability, proliferation, migration, and invasion, and EMT in cancers such as gastric cell carcinoma (Wang et al. 2021) and serous ovarian cancer (Ankasha et al. 2021). Furthermore, miR-200c-3p was reported to promote the cisplatin resistance in biliary tract cancer (Posch et al. 2021). However, the role of miR-200c-3p in human PTCs and its potential molecular mechanisms have not been widely studied up to now.

In this study, we explored how miRmiR-200c-3p participated in the PTC pathogenesis. We found that the miR-200c-3p expression was upregulated in human cell lines and PTC tissues. miR-200c-3p inhibition could suppress malignant biological behavior, including PTC cell colony formation, migration, proliferation, and invasion. We also found that ATPase sarcoplasmic/endoplasmic reticulum $\mathrm{Ca}^{2+}$ transporting 2 (ATP2A2) was a direct target of miR-200c-3p. The abnormal expression of miR-200c-3p significantly reversed the tumor-promoting effect of the miR-200c-3p overexpression in human PTC cells.

\section{Materials and Methods}

\section{Cell Culture}

The SV40-transfected human normal thyrocyte cell line Nthy-ori 3-1 (EK-Bioscience, Shanghai, China) was cultured in RPMI 1640 supplemented with $10 \%$ fetal bovine serum (FBS), 100 units/ml penicillin, and $100 \mu \mathrm{g} / \mathrm{ml}$ streptomycin (Chemical Book; Shanghai, China). Human papillary thyroid carcinoma cell lines TPC-1 and K1 were purchased from Sigma-Aldrich, and BCPAP cells were bought from Lianmai Biotech, Inc. (http://www.Imai-bio.com/; Shanghai, China). These thyroid cancer cells were cultured in Dulbecco's modified Eagle medium or nutrient mixture F-12 (DMEM/F12; 1:1, by volume) supplemented with 10\% FBS and penicillin/streptomycin (Gibco). All cells were kept in a humid incubator with $5 \% \mathrm{CO}_{2}$ at $37{ }^{\circ} \mathrm{C}$.

\section{Clinical Specimens}

PTC cancer tissues and adjacent normal thyroid tissues $(n=15$ pairs $)$ were collected from patients who underwent surgery at the Shaanxi Hospital of Traditional Chinese Medicine (Xi'an, China) between 2018 and 2019. The resected tissue 
was immediately frozen with liquid nitrogen and stored at $-80{ }^{\circ} \mathrm{C}$ before use. All patients were subjected to chemotherapy, radiotherapy, immunotherapy, or targeted therapy naïve before surgery. This study was approved by the Ethics Review Committee of the Shaanxi Hospital of Traditional Chinese Medicine. All patients or their guardians were asked to sign a written informed consent.

\section{Plasmids, miRNA Mimics, and Transfection}

The DNA coding sequence of ATP2A2 was cloned into a pcDNATM3.1 (+) mammalian expression vector (Invitrogen) with an empty vector as a negative control (NC). The miR-200c-3p mimic, inhibitor, and negative control (NC) mimic or inhibitor were chemically synthesized by Shanghai GenePharma Co., Ltd. (Shanghai, China). The sequences of the miR-200c-3p inhibitor or NC inhibitor were cloned into the GV280 vector (Biovector, Beijing) to generate PTC cells stably overexpressing the miR-200c-3p inhibitor or NC inhibitor. The PTC cells were incubated in 24-well plates at a density of $5 \times 10^{4} / \mathrm{ml}$ overnight and then transfected with the indicated miRNA mimics, inhibitors, and plasmids with Lipofectamine 3000 (Thermo Fisher Scientific, Inc.) in accordance with the manufacturer's instructions. They were further analyzed $48 \mathrm{~h}$ after transfection. Stable cell lines overexpressing the miR-200c-3p inhibitor or NC inhibitor were obtained through the limiting dilution method.

\section{Cell Proliferation and Colony Formation Assay}

The proliferation of K1 cells was examined with a CCK-8 assay (AccuRef Scientific, Xi' an, China) in accordance with the manufacturer's instructions. The absorbance at $450 \mathrm{~nm}$ of the sample in each well was determined using a microplate reader (BioRad Laboratories, Inc.; Hercules, CA, USA). In the colony formation assay, the K1 cells seeded in 12-well plates were transfected with the indicated miRNA inhibitors, mimics, and plasmids overnight. Then, 500 cells of each group were seeded into $35 \mathrm{~mm}$ dishes, and the medium was replaced with fresh complete DMEM/F12 every 2 days. After being cultured in an incubator at $37^{\circ} \mathrm{C}$ for 2 weeks, the cells were washed twice with phosphate-buffered saline (PBS) and fixed with $4 \%$ paraformaldehyde at room temperature for $10 \mathrm{~min}$. They were then stained with $0.1 \%$ crystal violet (Sigma-Aldrich) for $15 \mathrm{~min}$ and washed with tap water. This assay was repeated thrice with triplicate samples in each group. The dishes were observed and imaged under a microscope (XSP-13CC, Shanghai Yuguang Instrument; Shanghai, China). The average number of colonies was obtained from five randomly selected view fields.

\section{Wound Healing and Transwell Invasion Assay}

For wound healing assays, $1 \times 10^{6}$ cells/well of $\mathrm{K} 1$ cells were seeded in a six-well plate and maintained overnight to allow adequate adherence for the formation of a confluent monolayer. The cell layers were damaged using a sterile $200 \mu$ pipette 
tip and cleaned with a serum-free medium to eliminate dislodged cells. They were photographed immediately (time $0 \mathrm{~h}$ ) after being scratched and after being cultured with serum-free medium for $24 \mathrm{~h}$ (time $24 \mathrm{~h}$ ). For Transwell assays, Boyden chambers ( $8 \mu \mathrm{m}$ pore size, BD Biosciences; San Jose, CA, USA) coated with Matrigel (BD Biosciences) were used to evaluate the invasive potential of $\mathrm{K} 1$ cells. In simple terms, the cells were harvested $48 \mathrm{~h}$ after transfection and suspended in an FBS-free culture medium. Afterward, they were seeded into the upper chamber at a density of $1 \times 10^{5}$ in each well, and $200 \mu \mathrm{l}$ of the complete medium was added to the lower chamber. After $24 \mathrm{~h}$ of incubation, the medium was removed. The cells on the upper surface of the chamber were wiped off with a swab, and the invading cells on the lower surface of the membrane were fixed with $1 \%$ paraformaldehyde and stained with crystal violet (Sigma-Aldrich) at room temperature. The number of cells was counted under an inverted microscope with five random views for further analysis.

\section{Real-Time Quantitative PCR}

RNA samples were isolated with an RNA isolation kit (AccuRef Scientific) and an miRNeasy mini kit (Qiagen, Hilden, Germany; for miRNA). cDNA was synthesized using the PrimeScript ${ }^{\circledR}$ RT Master Mix Perfect real-time reagent kit (Takara Bio Inc.; for mRNA) or a universal tag with the miScript II RT kit (Qiagen; for miRNA). Real-time quantitative PCR (qPCR) was performed on an AB7500 RT-PCR instrument (Applied Biosystems, Foster City, CA, USA) in accordance with the standard protocol of the SYBR Green PCR kit (Toyobo, Osaka, Japan). Quantification was carried out through normalization to GAPDH (for mRNA) or U6 (for miRNA) with the $2^{-\Delta \Delta C T}$ method. The primers for qPCR analysis are listed in Table $\mathrm{S} 1$.

\section{Western Blot Assay}

The cells were lysed with the radioimmunoprecipitation assay lysis buffer (RIPA; Sigma-Aldrich). Then, the lysates were centrifuged at $13,000 \times g$ at $4{ }^{\circ} \mathrm{C}$ for $10 \mathrm{~min}$, and the supernatants were harvested as protein samples. After being quantified with a BCA protein quantification kit (AccuRef Scientfic), the protein samples were separated using $10 \%$ sodium dodecyl sulfate-polyacrylamide gel electrophoresis and transferred to polyvinylidene difluoride (PVDF) membranes. The PVDF membranes were then blocked with Tris-buffered saline supplemented with $0.1 \%$ Tween ${ }^{\circledR}$ 20 detergent buffer containing 5\% nonfat dried milk at room temperature for $1 \mathrm{~h}$. They were incubated with primary antibodies at $4{ }^{\circ} \mathrm{C}$ overnight. The anti-ATP2A2 antibody (Cat. No. PB0279) and the antibody for the loading control $\beta$-actin (Cat. No. BM0627) were purchased from Boster Biological Technology Co., Ltd. (California, USA). After binding to secondary antibodies, namely, horseradish peroxidase (HRP)-conjugated anti-rabbit IgG (cat. no HS101, TransGen Biotech, Inc.) or anti-mouse IgG (cat. no. HS201, TransGen Biotech, Inc.), the protein bands on the membranes were visualized using an enhanced chemiluminescence kit (AccuRef Scientific). The expression levels of the targeted proteins were quantified through densitometry. 


\section{Xenograft Mouse Model}

Male nude mice (6-8 weeks old; Charles River Laboratories, Beijing, China) were acclimated in a specific-pathogen-free facility at the Experimental Animal Center of the Shaanxi Hospital of Traditional Chinese Medicine for at least 1 week. Stable K1 cells expressing the miR-200c-3p inhibitor or NC inhibitor were subcutaneously injected into the right flanks of the randomly selected nude mice at a dose of $1 \times 10^{6}$ cells per mouse. The tumor size was measured every 4 days. The tumor volume $(V)$ was monitored once every 4 days and calculated using the following formula: $V=a b^{2} / 2$, where $a$ is the long diameter, and $b$ is the short diameter. The mice were sacrificed 18 days after the tumor cells were inoculated. Then, the tumors were isolated, weighed, imaged, and analyzed. All animal experiments were performed in accordance with the Guide for the Care and Use of Laboratory Animals (NRC 2011). The animal experimental procedures were approved by the Institutional Animal Care and Use Committee of Shaanxi Hospital of Traditional Chinese Medicine.

\section{Immunohistochemistry Staining}

The tumor tissues from xenograft mice were deparaffinized and rehydrated in serially graded ethanol solutions. Antigens were retrieved by heating the sections in $\mathrm{pH}$ 6.0 citric buffer in a microwave oven for $5 \mathrm{~min}$. After being washed with PBS and subsequently blocked with $3 \% \mathrm{H}_{2} \mathrm{O}_{2}$, the sections were incubated with rabbit-anti Ki-67 antibody (cat. No. ab15580, Abcam, Cambridge, MA) at $4{ }^{\circ} \mathrm{C}$ overnight. They were further washed with PBS and incubated with HRP-conjugated secondary antirabbit monoclonal antibody (Cat. No. HS101, TransGen Biotech, Inc.) at room temperature for $40 \mathrm{~min}$. They were washed again with PBS, stained with $0.5 \%$ diaminobenzidine, and counterstained with Mayer's hematoxylin. The mounted slides were observed under a microscope (Axio Scan.Z1, ZEISS; Jena, Germany).

\section{Target Prediction and Luciferase Reporter Assay}

The mRNA targets of miR-200c-3p were predicted with a combination of public algorithms, namely, Targetscan (http://www.targetscan.org/), microT (https://bio. tools/DIANA-microT), and picTar (https://pictar.mdc-berlin.de/). The predicted binding sites in the wild-type 3' UTR of the mRNA of ATP2A2 and the corresponding mutated binding region were subcloned into a luciferase-expressing vector pGL3. The K1 cells seeded in 24-well plates at a density of $1 \times 10^{5}$ cells/well were transfected with $100 \mathrm{nM}$ miR-200c-3p mimic or NC mimic, together with $100 \mathrm{ng}$ of the plasmid-expressing wild-type or mutated 3' UTR of the mRNA of ATP2A2. The cells were collected $48 \mathrm{~h}$ after transfection, and the luciferase activity was analyzed with a dual-luciferase reporter assay system (Promega; Madison, WI, USA) in accordance with the manufacturer's protocols. 


\section{Statistics}

Data were presented as mean \pm standard deviation and statistically analyzed using GraphPad Prism 8 (San Diego, CA). Differences between means were examined with Student's $t$ test or ANOVA. Pearson correlation was also conducted. Survival rates were evaluated using the Kaplan-Meier method with a log-rank test. Data with $P<0.05$ were considered statistically significant.

\section{Results}

\section{miR-200c-3p is Highly Expressed in PTC Cells, and Its High Expression predlcts the Poor Prognosis of Patients with PTC}

To explore the role of miRNAs in the development of PTC, we performed qPCR to evaluate the expression levels of 10 miRNAs in 15 paired PTC tissues and adjacent normal tissues. Among the candidate miRNAs, miR-200c-3p demonstrated more than threefold of upregulated expression in the malignant samples compared with that of the benign controls (Fig. 1A). The analysis of the THCA-TCGA database also revealed that the expression of miR-200c-3p was significantly higher in the primary PTC tissues than in the normal tissues (Fig. 1B). Moreover, we evaluated the expression level of miR-200c-3p in some typical human PTC cell lines. The expression levels of miR-200c-3p in TPC-1, K1, and BCPAP cells were more than that of the control Nthy-ori 3-1. Among these cells, K1 cells displayed the highest level of miR-200c-3p (Fig. 1C). Therefore, they were selected for further functional studies. Furthermore, we categorized the patients with PTC into two groups using the moderate expression level of miR-200c-3p (moderate $=4.946$ ) in tumor tissues as a cutoff value. We found that the survival of patients with PTC with a high miR200c-3p expression was significantly worse than that of patients with PTC with a low miR-200c-3p expression (Fig. 1D). Therefore, miR-200c-3p participated in the development of PTC.

\section{miR-200c-3p Inhibition Suppresses the Progression of PTC Cells In Vitro And In Vivo}

A high miR-200c-3p expression in PTC cells suggests that miR-200c-3p is a potential oncogenic gene that promotes PTC progression. To validate this hypothesis, we examined the effects of miR-200c-3p inactivation on the following malignant behaviors of human PTC cells: cell proliferation, colony formation, migration, and invasion. In Fig. 2A, the expression of miR-200c-3p in the K1 cells transfected with miR-200c-3p inhibitors was substantially downregulated. The proliferation of the $\mathrm{K} 1$ cells with inactivated miR-200c-3p was significantly compromised, especially at 48 and $72 \mathrm{~h}$ during the 3-day culture period, compared with that of the control K1 cells (Fig. 2B). The colony formation assay revealed that the number of colonies of the $\mathrm{K} 1$ cells transfected with miR-200c-3p mimics significantly reduced (Fig. 2C). 
A

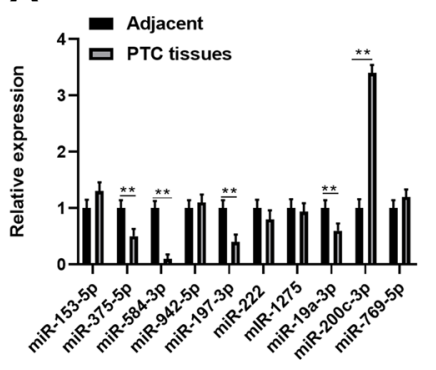

C

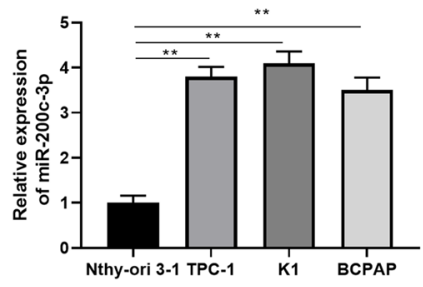

B
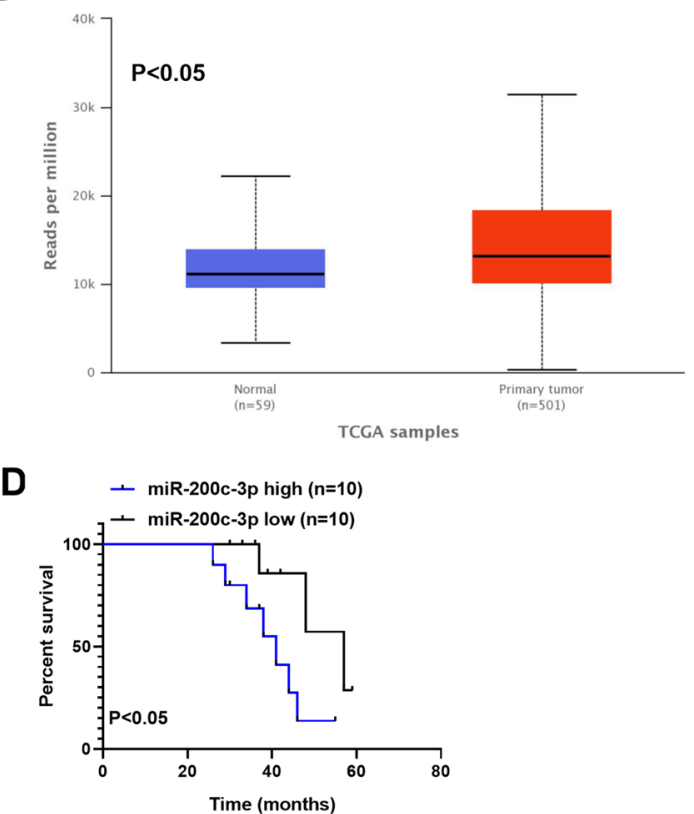

Fig. 1 miR-200c-3p was overexpressed in papillary thyroid carcinoma (PTC) and associated with poor prognosis of patients with PTC. A The expression levels of the indicated miRNAs in PTC tissues and paired adjacent normal tissues $(n=15)$ were evaluated through real-time quantitative PCR (qPCR). B The expression levels of miR-200c-3p in the TCGA-THCA data set (https://portal.gdc.cancer.gov/proje cts/TCGA-THCA) were retrieved and graphed; $n=59$ for normal tissues and $n=501$ for primary cancer tissues. C The relative expression levels of miR-200c-3p in the immortalized human thyroid follicular epithelial cell line Nthy-ori 3-1 and three human papillary thyroid carcinoma cell lines, namely, TPC1 , K1, and BCPAP, were quantitated through qPCR; $n=3$ for each group. Their differences were analyzed with Student's $t$ test in GraphPad Prism 8. D Kaplan-Meier analysis of the association between the expression of miR-200c-3p and the overall survival rate of patients with PTC; $n=10$ for the miR200c-3p-high group and $n=10$ for the miR-220c-3p-low group. $* P<0.05$ and $* * P<0.01$ between the indicated groups

The wound healing assay also demonstrated that miR-200c-3p inhibitors remarkably weakened the migration ability of the K1 cells (Fig. 2D). Similarly, the Transwell invasion assay confirmed that the invasion ability of the K1 cells with inactivated miR-200c-3p was significantly suppressed (Fig. 2E). Therefore, miR-200c-3p had an oncogenic role in PTC progression in vitro.

We also established a xenograft mouse model to evaluate the effect of miR$200 c-3 p$ inactivation on the progression of PTC cells in vivo. We found that miR200c-3p inhibition significantly suppressed the growth of xenografted K1 cells in nude mice. The tumor volume in the miR-200c-3p inhibitor group was significantly smaller than that in the NC group during the 17-day observation period after tumor inoculation (Fig. 3A). This finding was supported by the shrunken tumor sizes (Fig. 3B) and the significant reduction of tumor weight in the miR-200c-3p inhibitor group (Fig. 3C). In addition, we confirmed that the miR-200c-3p expression of 

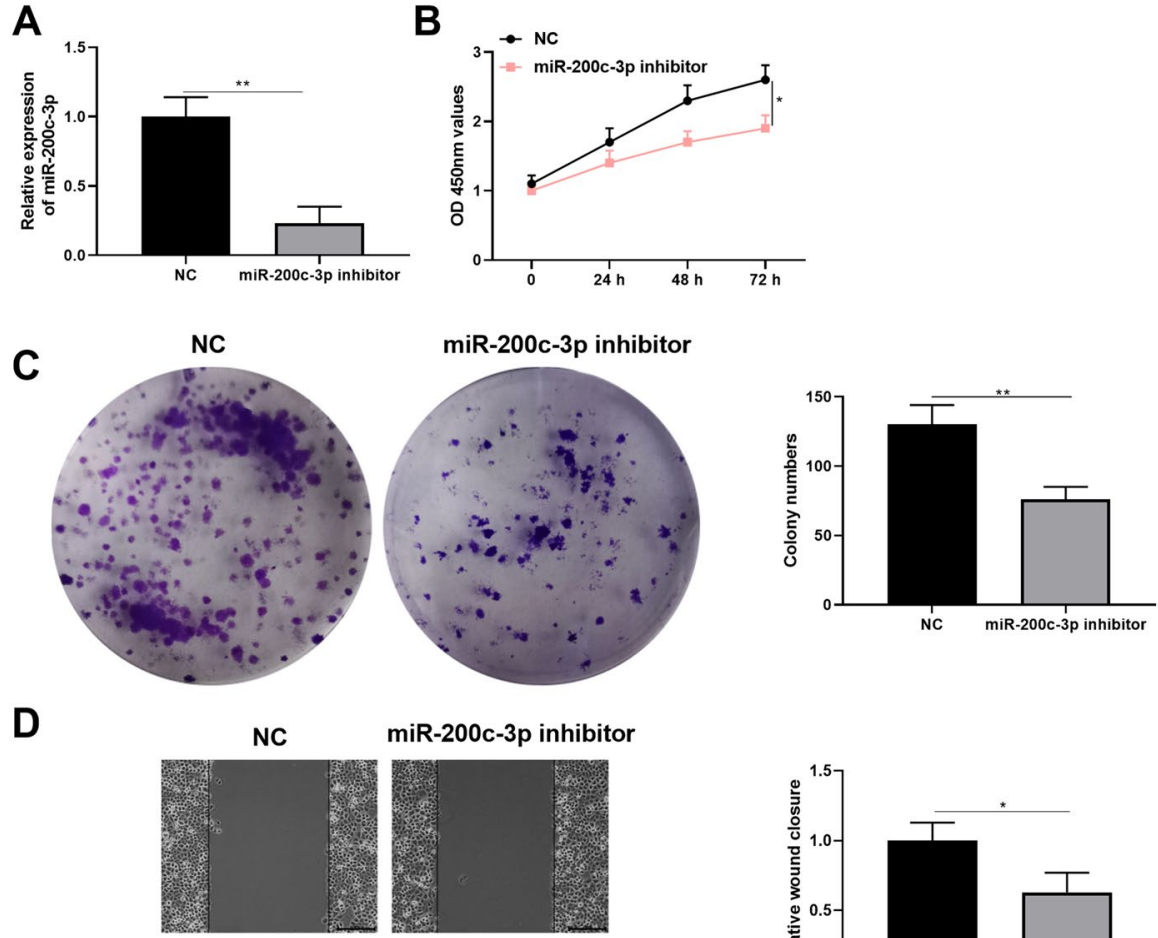

miR-200c-3p inhibitor

E
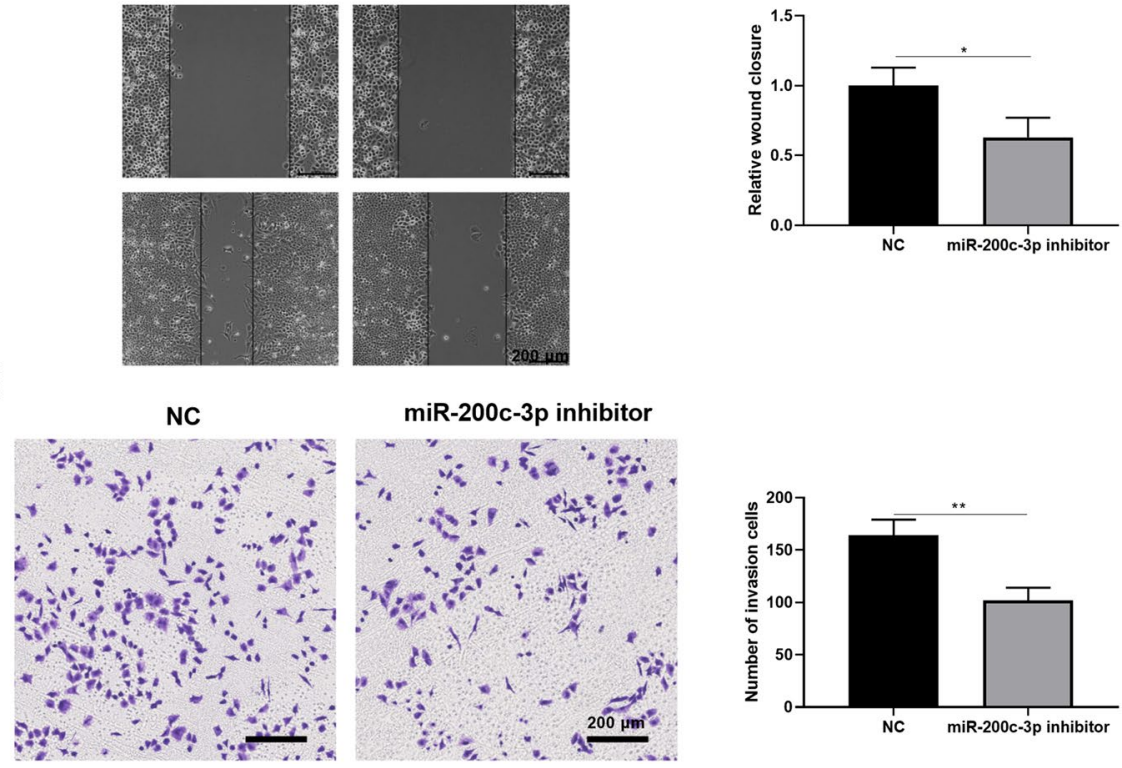

miR-200c-3p inhibitor
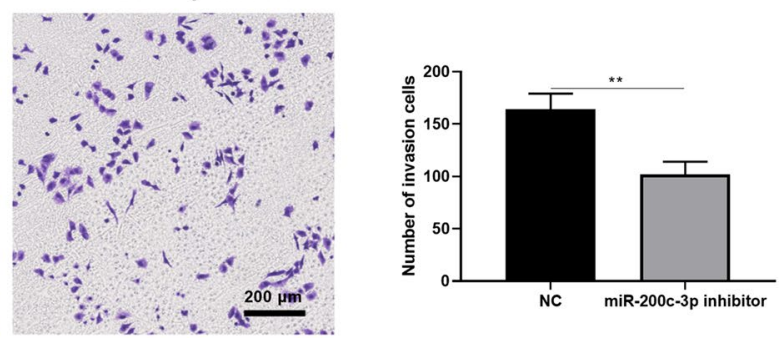

Fig. 2 miR-200c-3p inhibition suppressed the proliferation, migration, and invasion of PTC cells in vitro. A K1 cells were transfected with the miR-200c-3p inhibitor or negative control (NC) and harvested $48 \mathrm{~h}$ after transfection; the expression levels of miR-200c-3p were then determined through qPCR; $n=3$ for each group. B The proliferation of $\mathrm{K} 1$ cells transfected with the miR-200c-3p inhibitor or NC was determined via CCK-8 assays; $n=3$ for each group at each time point. $\mathbf{C}$ The colony formation ability of $\mathrm{K} 1$ cells after the indicated transfection was evaluated. Representative images were shown, and the number of colonies in each visual field was calculated; $n=5$ for each group. D Wound healing assay was performed to evaluate the migration ability of $\mathrm{K} 1$ cells after the indicated transfection. Representative images at 0 and $24 \mathrm{~h}$ were shown, and the relative wound closure was summarized; $n=3$ for each group. $\mathbf{E}$ Transwell invasion assay was conducted to evaluate the invasion ability of $\mathrm{K} 1$ cells after the indicated transfection. Representative images were shown, and the number of invading cells per visual field was summarized; $n=5$ for each group. $* P<0.05$ and $* * P<0.01$ between the indicated groups. Their differences were analyzed with Student's $t$ test in GraphPad Prism 8 

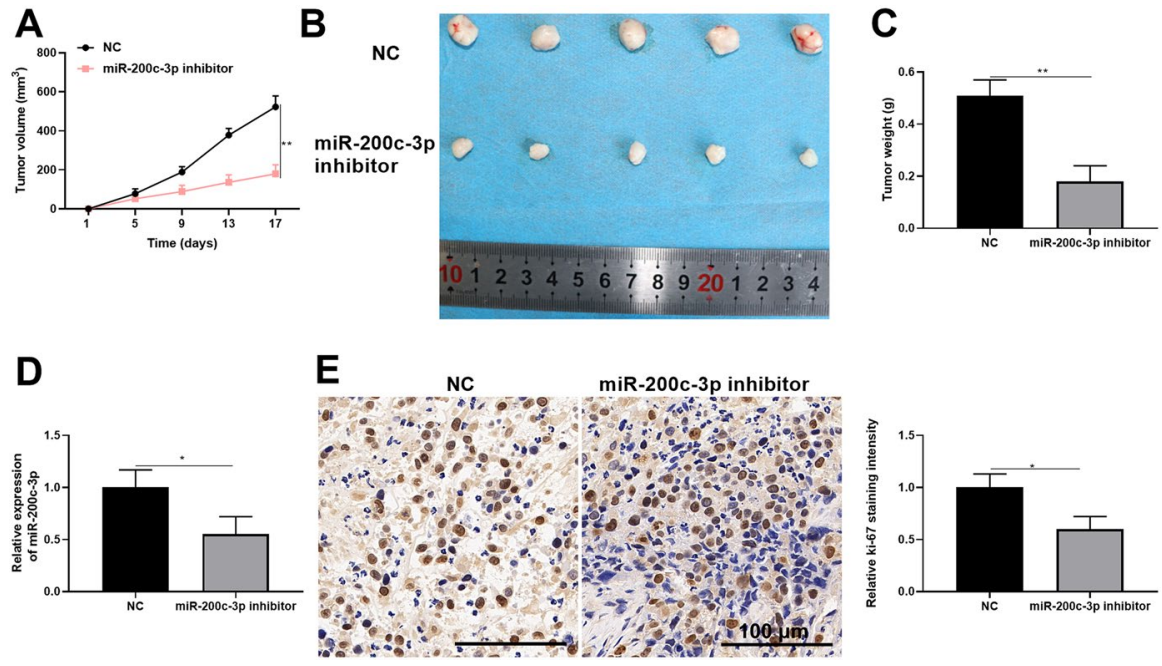

Fig. 3 miR-200c-3p inhibition suppressed the progression of PTC cells in vivo. A-E Stable K1 cells overexpressing the miR-200c-3p inhibitor or NC were injected subcutaneously into the right flank of nude mice $\left(1 \times 10^{6}\right.$ cells/mouse; $n=5$ for each group) to establish a human PTC xenograft model. Tumor volume was measured every 4 days, and tumor tissues were collected 18 days after tumor inoculation. The tumor growth curve (A) for 18 days and the tumor image $(\mathbf{B})$ and tumor weight $(\mathbf{C})$ on day 18 were summarized. D The expression levels of miR-200c-3p in tumor tissues on day 18 were summarized. E Tumor cell proliferation was determined in tumor tissues on day 18 through Ki-67 IHC. Representative IHC images were shown, and the relative Ki-67 staining intensity was summarized. $* P<0.05$ and $* * P<0.01$ between the indicated groups. Their differences were analyzed with Student's $t$ test in GraphPad Prism 8

the K1 cells markedly reduced (approximately 50\%) 18 days after inoculation compared with that of the control K1 cells (Fig. 3D). The IHC staining of Ki-67 revealed that the proliferation of the cells in the tumor tissue from the miR-200c-3p inhibitor group significantly decreased compared with that in the tumor tissues from the control group (Fig. 3E). Thus, miR-200c-3p inhibition suppressed the progression of PTC cells in vitro and in vivo.

\section{ATP2A2 is a Downstream Target of miR-200c-3p in PTC Cells}

To further explore the molecular mechanisms underlying the tumor-promoting function of miR-220c-3p, we used public algorithms to predict the target genes based on the consensus binding sites of miR-220c-3p. We found that 31 genes were the common targets predicted by three online tools (Fig. 4A). We then selected 11 putative candidates with the highest scores and examined their expression levels in the $\mathrm{K} 1$ cells transiently transfected with miR-200c-3p mimic or NC. We observed that ATP2A2 was the most downregulated upon miR-200c-3p overexpression in K1 cells (Fig. 4B). As such, we selected ATP2A2 as the putative downstream target of miR-200c-3p and performed multiple experiments to validate their relationship. The sequence located at the $3^{\prime}$ UTR of the mRNA of ATP2A2 (position 500-506) was highly complementary 
A

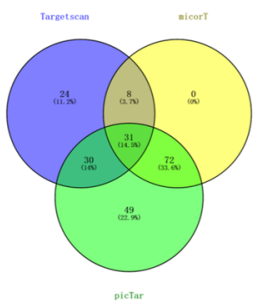

C

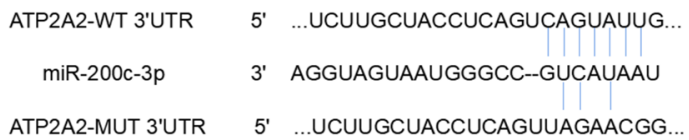

B
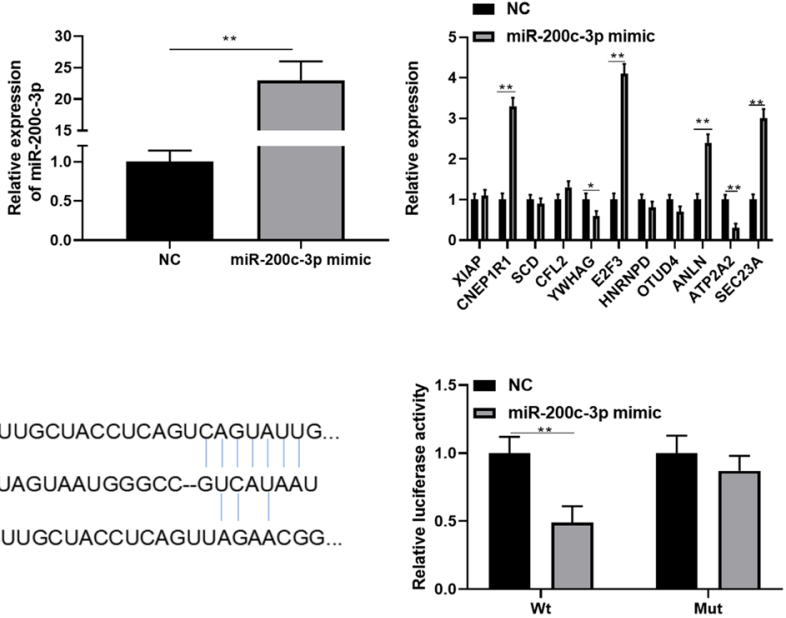

E

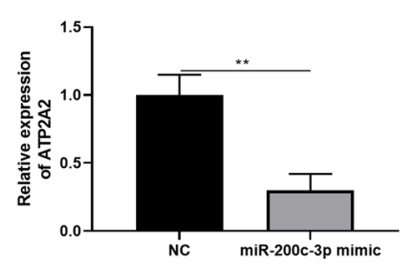

ATP2A2

$\beta$-actin
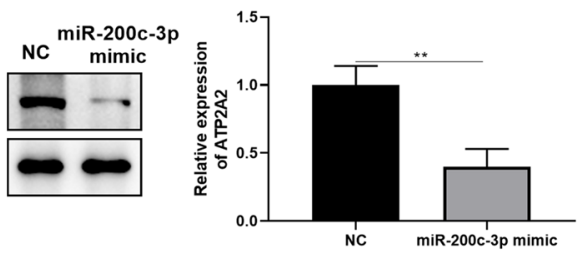

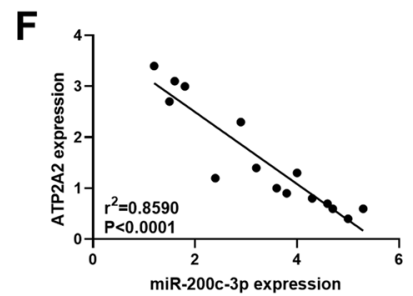

Fig. 4 miR-200c-3p regulated the ATP2A2 expression by binding to its $3^{\prime}$ UTR in PTC cells. A The Venn diagram showed the numbers of putative targets of miR-200c-3p, as calculated by three different online algorithms, including Targetscan, microT, and picTar. B K1 cells were transfected with an miR-200c-3p mimic or NC mimic and harvested $48 \mathrm{~h}$ after transfection. The expression levels of miR200c-3p (left) and 11 top candidate target genes (right) were determined through qPCR; $n=4$ for each group. C The diagrams illustrated the putative binding sites of miR-200c-3p and the corresponding wildtype (WT) and mutant (MUT) sites of the 3' UTR of mRNA of ATP2A2. The complete sequences of ATP2A2 and miRNA-200c-3p were predicted by TargetScan, picTar, and micorT. The binding site had a length of $7 \mathrm{bp}$ and was at positions 500-506 of the complete ATP2A2 sequence. The luciferase activity was detected in K1 cells after $48 \mathrm{~h}$ of the co-transfection of WT or MUT sites of the 3' UTR of ATP2A2, together with miR-200c-3p mimics or NC mimic; $n=4$ for each group. D, E The overexpression of miR-200c-3p downregulated the ATP2A2 expression in K1 cells. D The mRNA and E protein levels of ATP2A2 in K1 cells $48 \mathrm{~h}$ after the transfection of miR-200c-3p mimic or NC were determined through qPCR and Western blot, respectively. The representative images of bands were shown, and the relative levels of ATP2A2 were summarized from three independent experiments; $n=4$ for each group. F Pearson correlation analysis of the expression levels of miR-200c-3p and ATP2A2 in PTC tumor tissues $(n=15)$. $* P<0.05$ and $* * P<0.01$ between the indicated groups. Their differences were analyzed with Student's $t$ test in GraphPad Prism 8 
Fig. 5 ATP2A2 overexpression inhibited the proliferation, migration, and invasion of PTC cells in vitro. A-E K1 cells were transfected with an ATP2A2-expressing plasmid or an empty vector plasmid and harvested $48 \mathrm{~h}$ after transfection. A The mRNA level of ATP2A2 was determined through qPCR. B Cell proliferation for 3 days was examined via the CCK-8 assay. C The colony formation ability of K1 cells with and without ATP2A2 overexpression was determined. Representative images were shown, and the number of colonies was summarized. D The migration ability of K1 cells with and without ATP2A2 overexpression was determined via a wound healing assay. The representative images of wound initiation $(0 \mathrm{~h})$ and closure $(24 \mathrm{~h})$ were shown, and the relative wound closure was summarized. E The invasion ability of K1 cells with and without ATP2A2 overexpression was determined through Transwell experiments. Representative images were shown, and the number of invading cells was summarized; $n=4$ for each group. $* P<0.05$ and $* * P<0.01$, between the indicated groups. Their differences were analyzed with Student's $t$ test in GraphPad Prism 8

with the seed sequence of miR-200c-3p. We also designed the mutated 3' UTR of the mRNA of ATP2A2 to confirm the specific interaction between miR-200c-3p and wildtype 3' UTR of the mRNA of ATP2A2 (Fig. 4C). Luciferase assays demonstrated that the luciferase activity was significantly decreased only after the co-transfection of wildtype 3' UTR of ATP2A2 and miR-200c-3p mimics compared with that of the control groups transfected with NC mimics. However, it remained largely unchanged after the co-transfection of the mutated 3' UTR ATP2A2 and miR-200c-3p mimics in K1 cells (Fig. 4C). The mRNA (Fig. 4D) and protein (Fig. 4E) levels of ATP2A2 were significantly reduced in the $\mathrm{K} 1$ cells transfected with miR-200c-3p mimic compared with those in the cells transfected with the control miRNA mimic. Furthermore, the miR-200c-3p expression was inversely correlated with the ATP2A2 expression in PTC tumor tissues (Fig. 4F). This finding further supported the negative regulation of ATP2A2 expression by miR-200c-3p.

\section{ATP2A2 Overexpression Inhibits the Malignant Traits of PTC Cells In Vitro}

We next investigated the effects of the ATP2A2 expression on the malignant properties of K1 cells. After the transient transfection of the ATP2A2-expressing plasmid, the expression of ATP2A2 substantially increased compared with that of the cells transfected with the empty vector (Fig. 5A). During the 3-day culture period, the growth of the K1 cells with the ATP2A 2 overexpression was evidently slower in vitro, especially on days 2 and 3, than that of the control-transfected K1 cells (Fig. 5B). Similarly, the colony formation assay (Fig. 5C), the wound healing assay (Fig. 5D), and the Transwell invasion assay (Fig. 5E) revealed that the colony formation, migration, and invasion abilities of the K1 cells with the ATP2A2 overexpression were significantly compromised compared with those of the control K1 cells. Therefore, ATP2A 2 overexpression inhibited the malignant traits of PTC cells in vitro, implying that ATP2A2 functioned as a tumor-suppressor gene.

\section{ATP2A2 Overexpression Abolishes the Tumor-Promoting Effects of miR-200c-3p in PTC Cells In Vitro}

To further confirm the target relationship between miR-200c-3p and ATP2A2 in human PTC cells, we explored the effects of ATP2A2 overexpression on the 
A

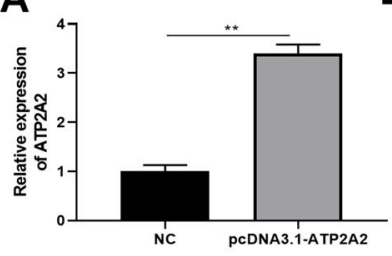

B

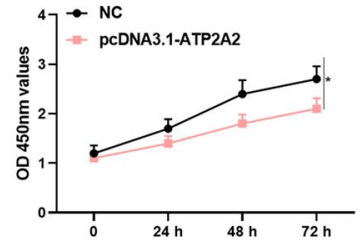

C

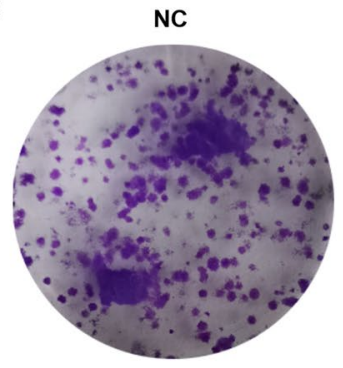

D
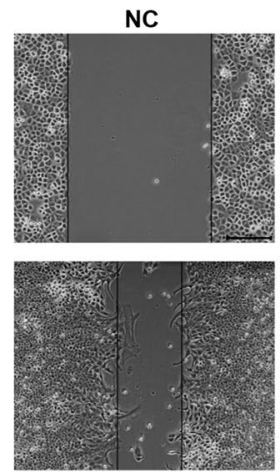

E

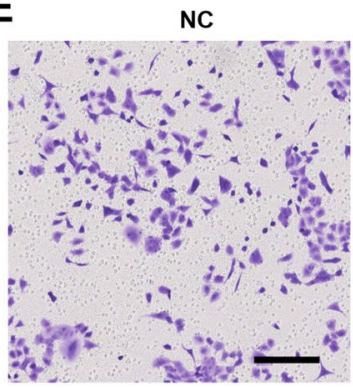

pcDNA3.1-ATP2A2

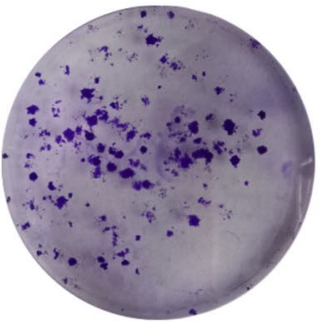

pcDNA3.1-ATP2A2
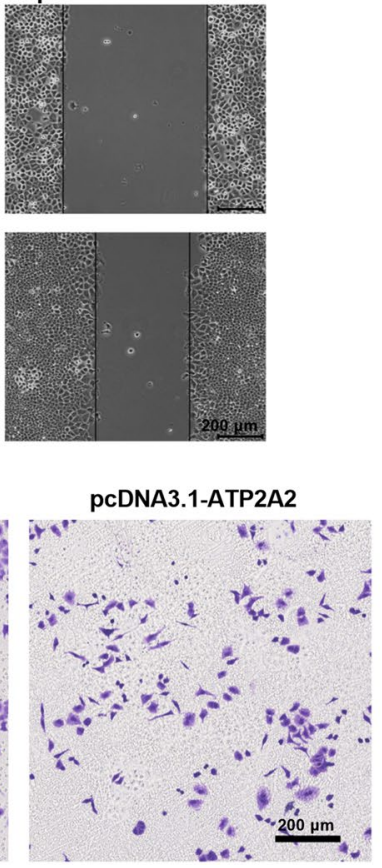
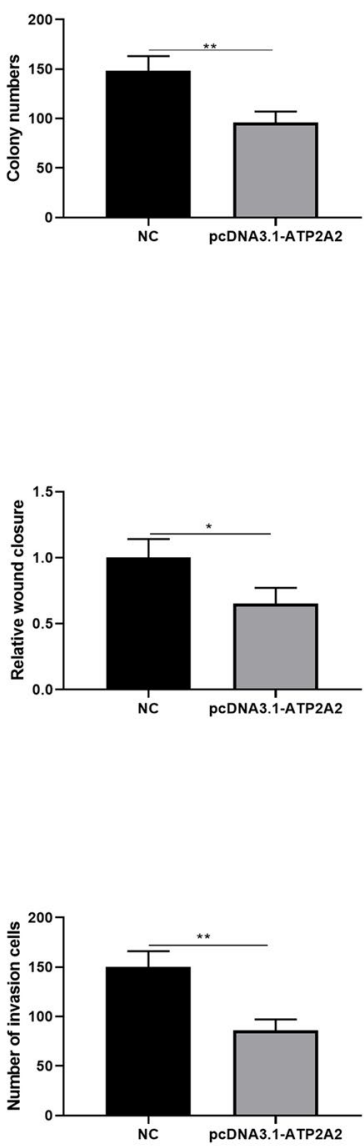

malignant behavior of the K1 cells transfected with miR-200c-3p mimic. The K1 cells were divided into four groups: control untransfected cells, cells transfected with the miR-200c-3p mimic alone, cells transfected with the ATP2A2-expressing plasmid alone, and cells transfected with the miR-200c-3p mimic and the ATP2A2expressing plasmid. We validated that miR-200c-3p mimic transfection significantly 
downregulated the ATP2A2 expression, whereas the additional transfection of the ATP2A2-expressing plasmid reversed the downregulated ATP2A2 expression compared with that of the control untransfected K1 cells (Fig. 6A). miR-200c-3p activation in the $\mathrm{K} 1$ cells significantly enhanced the cell proliferation, whereas further ATP2A2 overexpression reduced the cell growth. The group transfected with the miR-200c-3p mimic and the ATP2A2-expressing plasmid had comparable cell proliferation with that of the control group (Fig. 6B). Similarly, miR-200c-3p mimic alone significantly enhanced colony formation (Fig. 6C), migration (Fig. 6D), and invasion (Fig. 6E). Conversely, the additional ATP2A2 overexpression markedly compromised the effects of miR-200c-3p in promoting the malignant behaviors of K1 cells. Therefore, as a downstream effector of miR-200c-3p, ATP2A2 could reverse the tumor-promoting effects of miR-200c-3p in PTC cells.

\section{Discussion}

Although the 5-year survival of patients with PTC is over 95\%, some patients do not respond to radioactive iodine therapy and eventually suffer from cancer metastasis (Chen et al. 2009). As such, these patients have a poor prognosis, and their 10 -year survival decreases to approximately $10 \%$ (Hay et al. 2002). The oncogenesis and development of various human cancers, including PTCs (Hay et al. 2002), involve miRNAs, whose dysregulation is a common feature of human malignancies because they regulate the expression of oncogenes and tumor-suppressors (Hayes et al. 2014). Therefore, miRNAs may possess potential tumor diagnostic, prognostic, and therapeutic values in human PTCs.

In the present study, we observed the expression of 10 key tumor-associated miRNAs (miR-153-5p, miR-375-5p, miR-584-3p, miR-942-5p, miR-197-3p, miR222, miR-1275, miR-19a, miR-200c-3p, and miR-769-5p) in 15 PTC tumor tissues and paracancer normal tissues. We found that only the miR-200c-3p expression in PTC increased (more than threefold upregulated expression), speculating that miR200c-3p dysregulation might be the key to the malignant progression of PTC. Thus, we characterized the role of miR-200c-3p in controlling the malignant traits of PTC cells. We reported for the first time that miR-200c-3p was significantly upregulated not only in human PTC tumor tissues but also in cell lines. It also contributed substantially to the malignant behaviors of PTC cells, including proliferation, colony formation, migration, and invasion. For instance, miR-200c-3p inhibitor downregulated at least $20 \%$ of proliferation and about $40 \%$ of the number of plate clone/ invasion/migration of $\mathrm{K} 1$ cells in vitro. In addition, we validated the suppressing effect of miR-200c-3p inhibition on the in vivo growth of PTC cells using the K1 cell xenograft mouse model. Our results demonstrated that the miR-200c-3p inhibitor strongly inhibited not only the tumor volume and weight of the xenografted mice with K1 cells but also the expression of Ki67. We further identified ATP2A2 as a novel target of miR-200c-3p through bioinformatic analysis and luciferase reporter assay experiments. Moreover, the aberrant expression of ATP2A2 drastically reversed the tumor-promoting roles of miR-200c-3p mimics on the malignant 

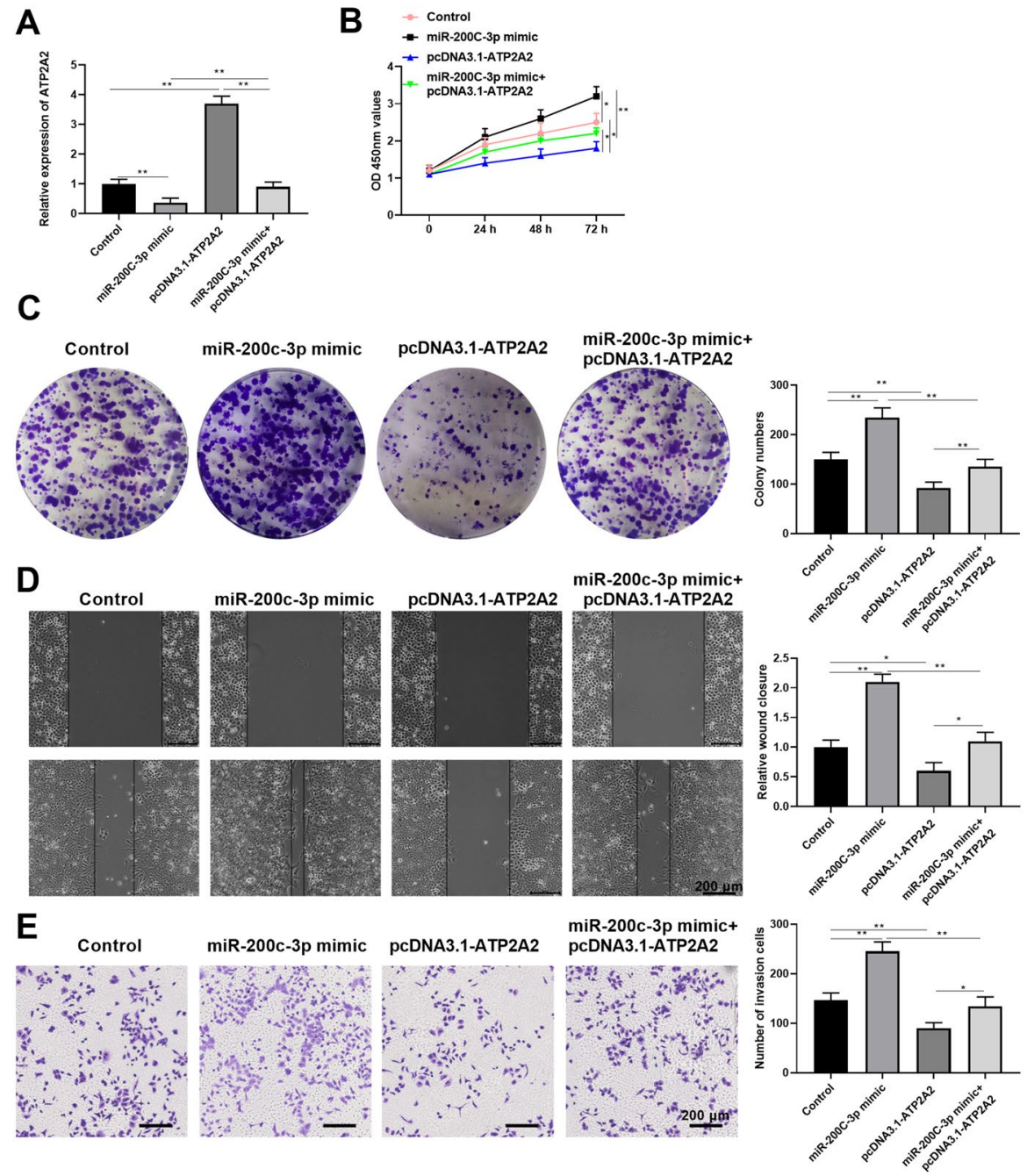

Fig. 6 ATP2A2 overexpression abrogated the promoting effects of miR-200c-3p on the malignancy of PTC cells. A-E K1 cells were left untransfected or transfected with miR-200c-3p alone, an ATP2A2expressing plasmid alone, or miR-200c-3p with the ATP2A2-expressing plasmid. They were harvested $48 \mathrm{~h}$ after transfection for subsequent experiments. A The relative level of ATP2A2 in K1 cells was determined through qPCR. B The proliferation of the indicated cells was determined during a 3-day culture period via the CCK-8 assay. $\mathbf{C}$ The colony formation ability, $\mathbf{D}$ migration ability, and $\mathbf{E}$ invasion ability of the indicated K1 cells were examined with $(\mathbf{C})$ a colony formation assay, $\mathbf{D}$ a wound healing assay, and $\mathbf{E}$ Transwell experiment, respectively. Representative images were shown, and the bar graphs were summarized from five independent experiments; $n=4$ for each group. Their differences were analyzed with Student's $t$ test in GraphPad Prism 8

behavior of human PTC cells. These findings suggested that the miR-200c-3p/ ATP2A2 axis could be a novel target for developing therapeutic strategies against PTCs. 
We found that miR-200c-3p was highly expressed in human PTC tissues and cell lines. Furthermore, its inactivation was associated with the suppressed malignant biological behavior of PTC cells. For instance, these cells had diminished proliferation, reduced colony formation ability, and compromised potentials of migration and invasion. These results demonstrated that miR-200c-3p functioned as an oncogenic factor in human PTCs. Additionally, the survival of patients with PTC with high miR-200c-3p expression levels in tumors was worse than that of patients with low miR-200c-3p expression levels. Similarly, the miR-200c expression level of ovarian cancer is much higher than that of normal ovarian tissues, and miR-200c expression is inversely associated with the advanced clinical stage and lymph node metastasis of ovarian cancer (Lu et al. 2014). miR-200c-3p inhibition ameliorates acute lung injury induced by $\mathrm{H} 5 \mathrm{~N} 1$ virus infection in vivo; moreover, nuclear factor- $\mathrm{\kappa B}-$ dependent upregulation of miR-200c-3p can reduce ACE2 levels, increase angiotensin II levels, and cause lung injury (Liu et al. 2017). The miR-200c-3p overexpression can significantly promote the proliferation activity, DNA replication, and invasion of endometrial cancer (EC) cells. miR-200c-3p remarkably suppresses phosphatase and tensin homolog deleted on chromosome ten (PTEN), which is a cancer suppressor, and activates the Akt activity by increasing phosphorylated Akt levels in EC cells (Liu et al. 2021). These studies have shown the destructive role of miR-200c-3p in diseases, including cancers and acute lung injury. However, miR200c-3p plays a tumor-suppressive role in multiple cancer types. For example, miR$200 c-3 p$ as a tumor-suppressor participates in the tumorigenesis and progression of various cancers, including human prostate cancer (Li et al. 2019a, b), human oral squamous cell carcinoma (Kawakubo-Yasukochi et al. 2018), renal cell carcinoma cells (Li et al. 2019a, b), and nephroblastoma cells (Li et al. 2019a, b). miR-200c-3p also contributes to the resistance of human gastric cancer cells to cisplatin (Jiang et al. 2017). A low miR-200c-3p level in tumors can predict a shorter overall survival of patients with pancreatic ductal adenocarcinoma (Zhuo et al. 2018). Therefore, the function of miR-200c-3p as a tumor-suppressive or tumor-promoting factor is most likely cell-context dependent and determined by cancer type. In addition, circulating miR-200c-3p is a potential marker of the diagnosis of metastatic disease in patients with breast cancer (Manzano et al. 2017). However, studies should determine whether the plasma level of circulating miR-200c-3p can be used to diagnose patients with PTC.

miRNAs exert their biological functions by directly regulating the expression of functional genes, which include tumor-suppressors and oncogenes (Banelli et al. 2017; Luo et al. 2015). In our study, we used multiple bioinformatic algorithms to predict the gene targets of miR-200c-3p and identified that the mRNA of ATP2A2 contained a highly conserved miR-200c-3p binding sequence in the $3^{\prime}$ UTR. Our subsequent luciferase assay and analysis on the expression levels further confirmed the targeting relationship between miR-200c-3p and ATP2A2 in human PTCs. Sarco (endo) plasmic reticulum $\mathrm{Ca}^{2+}$-ATPase isoform 2 (SERCA2), which is the protein product of $A T P 2 A 2$ and involved as a transporter to move $\mathrm{Ca}^{2+}$ from the cytosol to the endoplasmic reticulum lumen ( $\mathrm{Li}$ et al. 2017). Mutations in ATP2A2 cause Darier's disease in humans, an autosomal dominant skin disorder characterized by the loss of adhesion between epidermal cells and abnormal keratinization 
(Sakuntabhai et al. 1999). $\mathrm{Ca}^{2+}$ homeostasis is necessary to maintain the physiological functions of cells, so ATP2A2 mutation has been implicated in some malignancies. For example, phylogenetic changes in ATP2A2 may lead to the development of lung cancer and colon cancer, and the inactivation of ATP $2 A 2$ may be associated with cancer development as an early event (Korosec et al. 2006). ATP2A2 suppresses astrocytoma growth, and a high ATP2A2 expression in patients with diffuse astrocytic tumor is associated with better prognosis ( $\mathrm{Li}$ et al. 2017). However, the expression of ATP2A2 and its role in the oncogenesis of other cancer types have not been elucidated. Here, we confirmed that ATP2A2 had a lower expression level in PTC tissues than in normal tissues. More importantly, ATP2A2 was identified as a tumor-suppressor of PTC because its ectopic expression remarkably suppressed the malignant behaviors of PTC cells. For instance, it significantly compromised the potentials of cell proliferation, colony formation, migration, and invasion. Therefore, consistent with the report on the anti-tumor activity of ATP2A2 in astrocytoma (Li et al. 2017), ATP2A2 also functioned as a tumor-suppressor in human PTC.

This study had some strengths and limitations. It is the first to report the oncogenic role of miR-200c-3p in thyroid cancer and reveal the tumor-suppressor property of ATP2A2 in thyroid cancer. Although we demonstrated the apparent oncogenic role of miR-200c-3p in human PTCs in vitro and in an animal model, further studies should elucidate the use of miR-200c-3p as a cellular environmental limiting factor and determinant of oncogenes or tumor-suppressors. Moreover, a single miRNA may have multiple target miRNAs, which enable the involvement of a given miRNA in numerous biological processes. To fully clarify the tumor-promoting role of miR-200c-3p in the pathogenesis of human PTC, we recommend that further studies should be performed to screen the potential targets of miR-200c-3p other than ATP2A2, ZEB2, and FRS2.

In summary, miR-200c-3p acted as an oncogenic gene whose inactivation could suppress the malignant biological behavior of human PTC cells in vitro and in vivo. ATP2A2 was validated as a direct target gene of miR-200c-3p. Its overexpression remarkably reversed the tumor-promoting effects of miR-200c-3p mimics in human PTC cells. Therefore, the miR-200c-3p/ATP2A2 axis could be a new mechanism underlying the pathogenesis of human PTCs. It could be used as a basis for developing targeted therapeutics for human PTCs.

Supplementary Information The online version contains supplementary material available at https://doi. org/10.1007/s10528-022-10184-w.

Funding Funding was provided by Nature Science Foundation of Shaanxi Province (Grant Nos. 2019JM570 and 2020JQ-949) and also the Construction Project of the Traditional Chinese Medicine Academic Inheritance of the State Administration of Traditional Chinese Medicine (LPGZS2012-5).

\section{References}

Ambros V (2004) The functions of animal microRNAs. Nature 431(7006):350-355. https://doi.org/10. 1038/nature02871 
Ankasha SJ, Shafiee MN, Abdul Wahab N, Raja Ali RA, Mokhtar NM (2021) Oncogenic role of miR200c-3p in high-grade serous ovarian cancer progression via targeting the 3'-untranslated region of DLC1. Int J Environ Res Public Health. https://doi.org/10.3390/ijerph18115741

Banelli B, Forlani A, Allemanni G, Morabito A, Pistillo MP, Romani M (2017) MicroRNA in glioblastoma: an overview. Int J Genomics 2017:7639084. https://doi.org/10.1155/2017/7639084

Bartel DP (2004) MicroRNAs: genomics, biogenesis, mechanism, and function. Cell 116(2):281-297. https://doi.org/10.1016/s0092-8674(04)00045-5

Chen AY, Jemal A, Ward EM (2009) Increasing incidence of differentiated thyroid cancer in the United States, 1988-2005. Cancer 115(16):3801-3807. https://doi.org/10.1002/cncr.24416

Chen D, Zhang C, Chen J, Yang M, Afzal TA, An W, Xiao Q (2021) miRNA-200c-3p promotes endothelial to mesenchymal transition and neointimal hyperplasia in artery bypass grafts. J Pathol 253(2):209-224. https://doi.org/10.1002/path.5574

Coca-Pelaz A, Shah JP, Hernandez-Prera JC, Ghossein RA, Rodrigo JP, Hartl DM, Ferlito A (2020) Papillary thyroid cancer-aggressive variants and impact on management: a narrative review. Adv Ther 37(7):3112-3128. https://doi.org/10.1007/s12325-020-01391-1

Deng X, Wu B, Xiao K, Kang J, Xie J, Zhang X, Fan Y (2015) MiR-146b-5p promotes metastasis and induces epithelial-mesenchymal transition in thyroid cancer by targeting ZNRF3. Cell Physiol Biochem 35(1):71-82. https://doi.org/10.1159/000369676

Geraldo MV, Yamashita AS, Kimura ET (2012) MicroRNA miR-146b-5p regulates signal transduction of TGF- $\beta$ by repressing SMAD4 in thyroid cancer. Oncogene 31(15):1910-1922. https://doi.org/10. 1038/onc.2011.381

Ghafouri-Fard S, Shirvani-Farsani Z, Taheri M (2020) The role of microRNAs in the pathogenesis of thyroid cancer. Noncoding RNA Res 5(3):88-98. https://doi.org/10.1016/j.ncrna.2020.06.001

Hay ID, Thompson GB, Grant CS, Bergstralh EJ, Dvorak CE, Gorman CA, Goellner JR (2002) Papillary thyroid carcinoma managed at the Mayo Clinic during six decades (1940-1999): temporal trends in initial therapy and long-term outcome in 2444 consecutively treated patients. World J Surg 26(8):879-885. https://doi.org/10.1007/s00268-002-6612-1

Hayes J, Peruzzi PP, Lawler S (2014) MicroRNAs in cancer: biomarkers, functions and therapy. Trends Mol Med 20(8):460-469. https://doi.org/10.1016/j.molmed.2014.06.005

He H, Jazdzewski K, Li W, Liyanarachchi S, Nagy R, Volinia S, de la Chapelle A (2005) The role of microRNA genes in papillary thyroid carcinoma. Proc Natl Acad Sci U S A 102(52):19075-19080. https://doi.org/10.1073/pnas.0509603102

Jiang T, Dong P, Li L, Ma X, Xu P, Zhu H, Wang L (2017) MicroRNA-200c regulates cisplatin resistance by targeting ZEB2 in human gastric cancer cells. Oncol Rep 38(1):151-158. https://doi.org/10.3892/ or.2017.5659

Kawakubo-Yasukochi T, Morioka M, Hazekawa M, Yasukochi A, Nishinakagawa T, Ono K, Nakashima M (2018) miR-200c-3p spreads invasive capacity in human oral squamous cell carcinoma microenvironment. Mol Carcinog 57(2):295-302. https://doi.org/10.1002/mc.22744

Korosec B, Glavac D, Rott T, Ravnik-Glavac M (2006) Alterations in the ATP2A2 gene in correlation with colon and lung cancer. Cancer Genet Cytogenet 171(2):105-111. https://doi.org/10.1016/j. cancergencyto.2006.06.016

Li S, Feng Z, Zhang X, Lan D, Wu Y (2019a) Up-regulation of microRNA-200c-3p inhibits invasion and migration of renal cell carcinoma cells via the SOX2-dependent Wnt/ $\beta$-catenin signaling pathway. Cancer Cell Int 19:231. https://doi.org/10.1186/s12935-019-0944-5

Li T, Zhao P, Li Z, Wang CC, Wang YL, Gu Q (2019b) miR-200c-3p suppresses the proliferative, migratory, and invasive capacities of nephroblastoma cells via targeting FRS2. Biopreserv Biobank 17(5):444-451. https://doi.org/10.1089/bio.2019.0009

Li WQ, Zhong NZ, He J, Li YM, Hou LJ, Liu HM, Lu YC (2017) High ATP2A2 expression correlates with better prognosis of diffuse astrocytic tumor patients. Oncol Rep 37(5):2865-2874. https://doi. org/10.3892/or.2017.5528

Limaiem F, Rehman A, Mazzoni T (2021) Papillary thyroid carcinoma. StatPearls Publishing, Treasure Island

Liu Q, Du J, Yu X, Xu J, Huang F, Li X, Jiang C (2017) miRNA-200c-3p is crucial in acute respiratory distress syndrome. Cell Discov 3:17021. https://doi.org/10.1038/celldisc.2017.21

Liu Y, Cai X, Cai Y, Chang Y (2021) lncRNA OIP5-AS1 suppresses cell proliferation and invasion of endometrial cancer by regulating PTEN/AKT via sponging miR-200c-3p. J Immunol Res 2021:4861749. https://doi.org/10.1155/2021/4861749 
Lu YM, Shang C, Ou YL, Yin D, Li YN, Li X, Zhang SL (2014) miR-200c modulates ovarian cancer cell metastasis potential by targeting zinc finger E-box-binding homeobox 2 (ZEB2) expression. Med Oncol 31(8):134. https://doi.org/10.1007/s12032-014-0134-1

Luo JW, Wang X, Yang Y, Mao Q (2015) Role of micro-RNA (miRNA) in pathogenesis of glioblastoma. Eur Rev Med Pharmacol Sci 19(9):1630-1639

Manzano EN, Luengo-Gil G, Garcia-Garre E, Perez M, Ayala F (2017) Circulating miR-200c-3p as a marker of metastatic disease at diagnosis in breast cancer patients. J Clin Oncol 35(15 suppl):e12559-e12559

Mao Y, Jiang L (2021) MiR-200c-3p promotes ox-LDL-induced endothelial to mesenchymal transition in human umbilical vein endothelial cells through SMAD7/YAP pathway. J Physiol Sci 71(1):30. https://doi.org/10.1186/s12576-021-00815-Z

Pontemezzo E, Foglio E, Vernucci E, Magenta A, D’Agostino M, Sileno S, Limana F (2021) miR200c-3p regulates epitelial-to-mesenchymal transition in epicardial mesothelial cells by targeting epicardial follistatin-related protein 1. Int J Mol Sci. https://doi.org/10.3390/ijms22094971

Posch F, Prinz F, Balihodzic A, Mayr C, Kiesslich T, Klec C, Pichler M (2021) MiR-200c-3p modulates cisplatin resistance in biliary tract cancer by ZEB1-independent mechanisms. Cancers. https://doi. org/10.3390/cancers 13163996

Sakuntabhai A, Burge S, Monk S, Hovnanian A (1999) Spectrum of novel ATP2A2 mutations in patients with Darier's disease. Hum Mol Genet 8(9):1611-1619. https://doi.org/10.1093/hmg/8.9.1611

Siegel RL, Miller KD, Jemal A (2019) Cancer statistics, 2019. CA Cancer J Clin 69(1):7-34. https://doi. org/10.3322/caac. 21551

Sipos JA, Mazzaferri EL (2010) Thyroid cancer epidemiology and prognostic variables. Clin Oncol 22(6):395-404. https://doi.org/10.1016/j.clon.2010.05.004

Vecchia CL, Malvezzi M, Bosetti C, Garavello W, Bertuccio P, Levi F, Negri E (2015) Thyroid cancer mortality and incidence: A global overview. Int J Cancer J Int Cancer 136(9):2187-2195

Wang HY, Liu YN, Wu SG, Hsu CL, Chang TH, Tsai MF, Shih JY (2020) MiR-200c-3p suppression is associated with development of acquired resistance to epidermal growth factor receptor (EGFR) tyrosine kinase inhibitors in EGFR mutant non-small cell lung cancer via a mediating epithelialto-mesenchymal transition (EMT) process. Cancer Biomark 28(3):351-363. https://doi.org/10.3233/ CBM-191119

Wang Y, Lu K, Li W, Wang Z, Ding J, Zhu Z, Li Z (2021) MiR-200c-3p aggravates gastric cell carcinoma via KLF6. Genes Genom 43(11):1307-1316. https://doi.org/10.1007/s13258-021-01160-6

Xia L, Han Q, Chi C, Zhu Y, Pan J, Dong B, Sha J (2020) Transcriptional regulation of PRKAR2B by miR-200b-3p/200c-3p and XBP1 in human prostate cancer. Biomed Pharmacother 124:109863. https://doi.org/10.1016/j.biopha.2020.109863

Xing M (2013) Molecular pathogenesis and mechanisms of thyroid cancer. Nat Rev Cancer 13(3):184199. https://doi.org/10.1038/nrc3431

Zhuo M, Yuan C, Han T, Cui J, Jiao F, Wang L (2018) A novel feedback loop between high MALAT-1 and low miR-200c-3p promotes cell migration and invasion in pancreatic ductal adenocarcinoma and is predictive of poor prognosis. BMC Cancer 18(1):1032. https://doi.org/10.1186/ s12885-018-4954-9

Publisher's Note Springer Nature remains neutral with regard to jurisdictional claims in published maps and institutional affiliations.

\section{Authors and Affiliations}

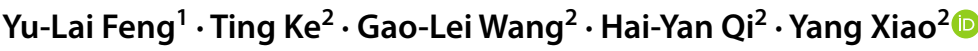

1 Department of Oncology, Lianyungang Hospital of Traditional Chinese Medicine, Chaoyang Middle Road, No. 160, Lianyungang 222004, China

2 Second Department of Endocrinology, Shaanxi Hospital of Traditional Chinese Medicine, Xihuamen, Lianhu district, No. 2, Xi' an 710003, China 